\title{
Ontology, Matter and Emergence ${ }^{1}$
}

\author{
Michel Bitbol \\ CREA/CNRS-Ecole Polytechnique, 1, rue Descartes, 75005 Paris France \\ e-mail:michel.bitbol@shs.polytechnique.fr
}

\section{Phenomenology and the cognitive science, forthcoming, 2007 (Online first : DOI 10.1007/s11097-006-9041-z) $<$ http://www.springerlink.com/content/606416j4rh8qq356/>}

\begin{abstract}
Ontological emergence" of inherent high-level properties with causal powers is witnessed nowhere. A non-substantialist conception of emergence works much better. It allows downward causation, provided our concept of causality is transformed accordingly.
\end{abstract}

\section{Foreword}

The thesis of the emergence of consciousness out of complex neurophysiological processes is commonplace (Freeman, 2001). Yet, it raises two major problems which are far from being correctly addressed. The first problem is a "category problem" (by due reference to G. Ryle's notion of "category mistake"). Emergence concerns properties, to wit features that are intersubjectively accessible, and that can then be described in a third-person mode. Saying that a property "consciousness" emerges from a complex network of interacting neurophysiological properties therefore misses a crucial point: that consciousness is no ordinary "property" in this sense, but rather a situated, perspectival, first-person mode of access. Any discourse about consciousness is thus bound to be first and second-personal in disguise (behind the curtain of a formally referential language). This problem of "radical situatedness" is discussed and developed in a recent paper of mine ${ }^{2}$. The second problem is an ontological problem, which concerns any case of emergence whatsoever, not only consciousness. If it is accepted that there are elementary properties (the properties of the putative basic constituants of the world), can there be genuine emergent properties? It is this second problem I wish to address in the present article, by way of a strategy of ontological deconstruction.

\footnotetext{
${ }^{1}$ A former version of this paper was intended as part of my contribution to the Mind \& Life meeting in Dharamsala (India) in october 2002 (in fact, only a small fraction of it was presented there). I thank all the participants, especially U. Goodenough, S. Chu, and P.L. Luisi for their cogent remarks, Arthur Zajonc for his empathic and lucid chairing, Adam Engle for his talent of organizer and warmth, Alan Wallace and Thupten Jinpa for their acute and creative translation, and Matthieu Ricard for his serene enthusiasm and friendship. Very special thanks are due to His Holiness the Dalai-Lama, who provided both his intellectual insight, and qualities which are hardly met in other conferences: a sense of high unity of purpose beyond the differences in standpoint, and a constant ethical "embodiment" of knowledge which caught us all.

${ }^{2}$ M. Bitbol, "Science as if situation mattered", Phenomenology and the Cognitive Science, 1, 181-224, 2002 ; also : M. Bitbol, Physique et Philosophie de l'Esprit, Flammarion, 2000
} 


\section{Introduction}

Are there truly new high level properties arising from nothing else than a large number of interacting low level components none of which possess these high level properties when taken in isolation? For example, can we say that life is a radically new property, or state, or process, arising (or emerging) from nothing else than a large number of interacting atoms and molecules none of which can be said to be alive when taken in isolation?

As one can hear from these sentences, the problem is formulated in heavily metaphysical terms. Those who raise it want to know whether there truly exist such emergent large-scale properties ; they want to know whether these largescale properties are more than just epiphenomena ; accordingly, they want to know whether they have or do not have the causal power of altering other (large-scale or micro-scale) properties. This metaphysical formulation of the problem of emergence is not surprising in view of its historical motivation. After all, the concept of emergence was invented in order to find a satisfactory compromise between two extreme ontologies. The first of these two ontologies is monist and materialist: it says that there exist nothing else than material elements and their properties. The second ontology is dualist: it says there are two substances or two realms of being: mind and matter, or life and inanimate matter. Emergentism aims at finding an ontological "middle course" ${ }^{3}$ between the monist materialist and the dualist ontologies. But being a middle path does not preclude showing a little bend towards one or the other of the two extremes. Emergentism comes very close to monist materialism if it takes the high level behavior as a superficial symptom, with no relevance whatsoever to the real physical processes taking place at the low level (this is the "supervenience" view). Conversely, emergentism comes closer to dualism when it tries to endow the emergent properties with some sort ontological consistence, and with causal powers of their own.

Obviously, it is the latter strong version of emergence that is interesting. But as we shall see, its exceedingly high ambition is also its major weakness. No convincing proof of there being genuinely, ontologically, emergent properties can be given. Even less so when one believes that the basic constituents of the world are little things (say the elementary particles) endowed with intrinsic properties. My conclusion will be that if there is a viable middle path, then it is definitely non-ontological; it presupposes a thorough criticism of ontological claims at every single level of knowledge.

To show this, I'll use a reductio ad absurdum reasoning. I'll suppose for a few paragraphs that the world is indeed made exclusively of particles endowed with intrinsic properties, and that the issue of emergence only bears on inherently existent high level properties. I'll then point out that in this case,

\footnotetext{
${ }^{3}$ C. Lloyd Morgan, Emergent evolution, London: Williams and Norgate, 1923, IV, §20, p. 115
} 
reductionism (namely the view according to which everything reduces to a bunch of lower level properties) easily wins the game. But then, I'll step back from any reifying conception and stress that in this case, the strongest argument by far comes from the anti-reductionist side.

Of course, a militant anti-reductionist thinker may be reluctant at this point, because she thinks : "If I weaken my ontological claims, isn't there the risk of leaving once again the reductionist the last word ? Can't the reductionist take advantage of my apparent concession and say that after all, her basic elements are the only real things in the world ?". So, I must reassure the antireductionist thinker immediately. My answer to her anxious question will be: "Not at all. By weakening your ontological claim, you are doing no harm whatsoever to your position; you are rather reinforcing it". For, if the message of quantum physics is taken seriously, the critique of reification concerns not only the high-level properties but also the low-level properties; not only the emerging properties, but also the properties of the so-called basic constituants of the world. The reductionist eventually loses the game, because her so-called "reduction basis" is as firm as quicksands, and because it proves quite easy in this case to put the emergent behavior on exactly the same footing as the socalled "elementary" entities and laws.

\section{The failure of strong emergence}

Now, let me come back to the reductio ad absurdum. Suppose that the world is indeed made exclusively of particles endowed with intrinsic properties. Then, it is clear by definition that, at the level of being, there is nothing else and nothing more in a high-level feature than the system of the underlying low-level properties. Nothing precludes that the high-level behavior manifests new and unexpected features with respect to the lowlevel; but under our very strong ontological assumptions, this is only a superficial appearance which does not support the further claim that the largescale features are really new, really autonomous, let alone that they have intrinsic existence. In other terms, ontological reductionism can easily accomodate emergence, provided this is only epistemological emergence ; provided this is only apparent emergence relatively to our limited faculties of knowledge. The appearance of new and autonomous features can easily be explained by ontological reductionists provided they assume that our experimental or perceptive analysis is coarse-grained. After all, if we use a poor instrument of study which cannot provide us with information below a certain scale, no wonder the large-scale behavior looks as if it were new and autonomous with respect to basic elements studied at a much higher resolution. In these matters, it is crucial "(...) to be clear about whether we are investigating a limitation of scientific description or something about actual 
physical processes in the world" ${ }^{\prime 4}$. A well-known case is that of thermodynamic properties, such as temperature, when they are seen from the standpoint of the kinetic theory of gases. If we accept that the basic constituents of a gas are little moving material bodies, then temperature is nothing else than a measure of their average state of disordered motion, as it is assessed by a very coarse and inaccurate instrument called the thermometer. Temperature looks as if it were a new and autonomous property, but it is so only relative to the thermometric technique, or relative to a poor large-scale statistical assessment.

True, there are situations that are not so easily sorted out. The example of temperature only concerns the most elementary sort of emergence : the one which comes closest to mere "supervenience". Other types of emergence, such as the "self-organizational", the "evolutionary", or the mental could prove more resistant to the reductionist's epistemological reading. When we see living beings displaying intentional behavior, or behavior apparently governed by purpose, we cannot easily figure out relative to what instrument this property of "intentionality" is defined. Haven't we put our fingers on a genuinely emergent property? No, says the reductionist. For it is not impossible to explain how an appearance of behavioral intentionality may arise in a non-intentional world made exclusively of particles with microproperties. The explanation, proposed by Daniel Dennett, has been called by him the "strategy of interpretation" or the "intentional stance". Even though, says Dennett, there are only particles and their micro-properties in the world; even though living bodies are ultimately bunches of particles moving here and there, the easiest way for $u s$ to predict their later motion is to interpret it as a purposeful, intentional action ${ }^{6}$. Therefore, behavioral intentionality is nothing new and nothing autonomous with respect to the basic constituents, but only a matter of convenience in the task of anticipating their future position whenever they belong to organized bodies. Here, what plays the role of the coarse-grained instrument relative to which behavioral intentionality looks as if it were a genuinely new feature, is the procedure of interpretation itself.

At this point, the subtle concessions made by the reductionist trigger an objection. In the very account given by the reductionist, the coarse-grained interpretation is not only another tool ; it is also a much better, much more efficient and economical tool, for that task of predicting phenomena. Sometimes it is even the only available tool for prediction. This remarkable

\footnotetext{
${ }^{4}$ T.W. Deacon, "The hierarchic logic of emergence: untangling the interdependence of evolution and selforganization ", in: B. Weber \& D. Depew (eds.), Evolution and learning: The Baldwin effect reconsidered, MIT Press, 2003.

${ }^{5}$ T.W. Deacon, "The hierarchic logic of emergence: untangling the interdependence of evolution and selforganization", loc. cit.

${ }^{6}$ Of course, this strategy of interpretation hides a crucial dialectic between third-person and first-person accounts: from behavior to intention, and then back again (by means of the usual strategy of projection) to intentional behavior. This issue of first-person standpoint will be briefly evoked in the conclusion.
} 
aptitude for anticipation of future behavior lends credibility to the assumption of genuine emergence of a new and autonomous high-level property called "intentionality". But beware: the reductionist is not defeated by this objection. She could reply (and I would agree with her on this particular point) that neither success of a method or a concept, nor optimal viability of an attitude, should be mistaken for a proof of the intrinsic reality of its object. Success of an action does not demonstrate the truth of the assumptions under which this action is performed; it only demonstrates ... their efficiency as guiding principles. Nietzsche rightly noticed that "a belief which is necessary to life may be wrong, in spite of this"

Much work has been done in the last decades to overcome the reductionist's resistance and to find better and better arguments in favor of the so-called "ontological emergence": emergence of truly existent high-level properties out of a basis of small interacting particles endowed with low-level properties. However, several philosophers, including myself, still remain unconvinced by these arguments. Let us list some of them.

The non-linearity of the equations ruling the elementary processes is the most abstract and the most general of these anti-reductionist arguments. The relevant point is that in non-linear processes, adding two causes does not yield addition of the two corresponding effects; it sometimes yields extreme amplification of the effects. A celebrated consequence is the so-called "deterministic chaos", in which non-linear yet deterministic interaction of a few components results in complex behavior of the system. The key point is that this complex behavior cannot be predicted from an initial state of the components, experimentally measured with limited accuracy. This is taken by some to mean that there is really more in the global behavior than in the individual processes. But actually, what it amounts to, is recognition that no matter how precisely you know the prevailing laws and the initial conditions of the components, you cannot derive with any reasonable precision the future behavior of the system. Here, even a very little inaccuracy in the knowledge of the initial conditions is enough to rule out any possibility of knowing in advance the final state. Little contingent events occurring at the beginning of the process or in the course of its history are more important for its future than the underlying elementary laws which are supposed to rule it.

No wonder that, in this case, reductionists have a strong argument in store. Here it is. If we want to say that we have witnessed the emergence of intrinsically existent high-level properties, we cannot content ourselves with showing that these high-level properties are not predictable in practice by means of a restricted knowledge of the initial conditions and of the underlying deterministic laws of (classical) dynamics. For, once again, this only yields methodological or epistemological emergence, not ontological emergence. It

\footnotetext{
${ }^{7}$ F. Nietzsche, Wille zur Macht, Kröners Taschenausgabe, Alfred Kröner, 1956, XVI §483
} 
can well be that the events which are not predictable by finite beings turn out to be predictable in principle. But, says the reductionist, what we want to address in this case is an issue of being, not an issue of knowledge; an issue of principles, not of practical limitation. According to her, the basic laws and constituents are and remain the low-level ones, and no remark about the sensitivity of the dynamics to initial conditions can weaken this fact.

In order to meet this strong objection, based on a reifying understanding of elementary constituents and laws, anti-reductionists often tended to be more radical in their own reifying assumptions, in order to counterbalance the micro-reifications of the reductionists. Some anti-reductionist thinkers thus suggested that chaos has to be taken very seriously as a complete dismissal of the laws of classical dynamics, including in the non-relativistic and nonquantum domain. They claimed that the laws of classical dynamics are only an approximation of more fundamental probabilistic laws. The real laws of nature being intrinsically probabilistic, any outcome, including emergent properties, would be autonomous with respect to its putative microscopic antecedents. The connection between the low level and the emergent level would be lost not due to imperfect knowledge of initial conditions, but rather due to real absence of any such connection. This would give strong support to the idea of a genuine or ontological emergence. But this proposal has not reached a general consensus. The suggestion of abandoning the laws of Newtonian dynamics in the non-relativistic and non-quantum domain looks too speculative. More seriously, ascribing an ultimately "fundamental" status to the postulated probabilistic law is not at all warranted; not any more than ascribing a "fundamental" status to the usual deterministic laws. Which one is fundamental with respect to the other is completely undecidable. Nothing can afford a criterion of choice. For it proves just as easy to derive the first type of law from the second (by means of the law of large numbers) as the other way around (by means of the formalism of "deterministic chaos"). But when one is interested in ontological questions, it is crucial to decide which are the real basic "real" laws of nature. One cannot rest content with statements of undecidability.

Yet another argument in favor of true ("ontological") emergence was given recently. This argument is quite independent on any discussion on the nature of the basic law which rule the low-level constituents; it rather concentrates on the high level. According to its proponents, even though we are unable to predict the high level behavior of a system by means of the laws that rule its low-level constituents, we can make some sort of predictions by using what Stuart Kauffmann calls the "laws of complexity", which hold at the high level. These "laws of complexity" are valid irrespective of the micro-laws of the underlying low level. They can be implemented on many different layers of "basic" elements, including the incredibly simple discrete algorithm used by Steven Wolfram. Or in other terms "The same (high level) topology can be 
differently instantiated" 8 . Therefore, since the lower level is irrelevant, there seems to be a very powerful reason to believe in the emergence of a genuine higher layer of autonomous properties.

However, this latter argument is undermined by two objections :

(i) The laws of complexity only rule the relation between classes of highlevel behaviors, not between individual high-level configurations. Therefore, the laws of complexity give one no right to say that each high-level configuration taken individually has a real effect by itself. But this kind of individual real efficacy is exactly what is needed to grant autonomy and ontological status to high-level configurations.

(ii) It may well be that the relative success of the laws of complexity has no further significance than the success of Dennett's "intentional stance" to predict the behavior of an intelligent living being : it is more economical than any other strategy; it is also more unified because it assumes only one explanatory scheme for the high level instead of providing as many explanatory schemes as there are lower levels on which it can be implemented. But, once again, economy is not enough to claim substantiality of the postulated high level properties. In an ontological debate we are concerned by what an ideal predictor could do with the micro-laws, not by what we, finite human beings, must be content with.

\section{Emergence and quantum mechanics}

Today, this series of negative arguments against "ontological emergence" is taken quite seriously by philosophers. Some of them ${ }^{9}$ concluded that the only plausible candidate for true emergence occurs in Quantum Mechanics. The candidate is nothing else than non-separability. This is not so surprising. After all, when, in the early days of Quantum Mechanics, Schrödinger discovered non-separability, he characterized it in terms which strongly evoke emergence : "Best possible knowledge of a whole does not necessarily include (best possible knowledge of) its parts. (...) The whole is in a definite state, the parts taken individually are not" ${ }^{\prime 0}$. These early remarks were recently developed in the context of the discussion on emergence. A fine-tuned study showed that, in quantum physics, the usual difference between additivity and non-additivity of properties must be replaced by a contrast between : (i) factorizability in a tensor product of states or Hamiltonians, and (ii)

\footnotetext{
${ }^{8}$ T.W. Deacon, “ The hierarchic logic of emergence: untangling the interdependence of evolution and selforganization ", loc. cit.

9 Michael Silberstein \& John McGeever, "The Search for Ontological Emergence", The philosophical Quarterly, 49, 182-200, 1999; P. Humphreys, "How Properties Emerge ”, Philosophy of Science, 64, 1-17, 1997; F.M. Kronz \& J. T. Tiehen, “Emergence and Quantum Mechanics ”, Philosophy of Science, 69, 324347, 2002.

${ }^{10}$ E. Schrödinger, “The Present Situation of Quantum Mechanics”, 1935 ; English Translation in Section I.11 of Quantum Theory and Measurement, J.A. Wheeler and W.H. Zurek, eds., Princeton university Press, 1983.
} 
entanglement of states or interaction Hamiltonians ${ }^{11}$. In the same way as, in classical physics, non-additivity yields emergence of (apparent or real) new features on a permanent ground of elementary parts, in quantum physics nonfactorizability yields emergence of (allegedly real) new features wherein the initial elements are so to speak resorbed. It was then pointed out that this quantum situation, in which the state of the whole determines the state of the parts rather than the other way around, utterly contrasts with the usual assumption of "supervenience" according to which the state of the parts determine the state of the whole. With this idea of Quantum Mechanics in mind, a stimulating view of "ontological" emergence was formulated. Assuming a hierarchy of levels of properties, P. Humphreys ${ }^{12}$ suggested that properties at the $\mathrm{j}$-th level can arise from the "fusion" of two or more properties at the $\mathrm{i}$-th level (where $\mathrm{i}<\mathrm{j}$ ). Here "fusion" is taken literally, since it means "disappearance" of what is fused within a new entity. This being granted, the lower (i-th level) properties can have no causal effect of their own, because they no longer exist after the fusion. The only properties that have a direct mutual causal connection are the fused properties of high (j-th) level. If some causal connection between two lower (i-th level) properties nevertheless occurs, it does so through the mediation of a complex process involving the upper ( $\mathrm{j}$-th) level. An example of this complex multi-level process is the following:

(a) Two properties $\mathrm{p}_{\mathrm{i} 1}$ and $\mathrm{p}_{\mathrm{i} 2}$ of the $\mathrm{i}$-th level "fuse", thus generating a $\mathrm{j}$-th level property $\mathrm{P}_{\mathrm{j}}$ (emergence);

(b) The $\mathrm{j}$-th level property $\mathrm{P}_{\mathrm{j}}$ undergoes a causal process and transforms into $\mathrm{P}_{\mathrm{j}}$ according to a law-like evolution;

(c) the transformed $\mathrm{j}$-th level property $\mathrm{P}_{\mathrm{j}}^{*}$ splits into a pair of new properties $\mathrm{p}^{*}{ }_{\mathrm{i} 1}$ and $\mathrm{p}^{*}{ }_{\mathrm{i} 2}$ of the $\mathrm{i}$-th level (submergence).

Within this frame of thought, the only genuine causal process is the one that takes place at the higher $(\mathrm{j}$-th) level of organization, whereas the transformation of $\mathrm{p}_{\mathrm{i} 1}$ and $\mathrm{p}_{\mathrm{i} 2}$ into $\mathrm{p}^{*}{ }_{\mathrm{i} 1}$ and $\mathrm{p}^{*}{ }_{\mathrm{i} 2}$ generates a mere appearance of causality at the i-th level. Here, the distribution of the qualifyers "apparent" and "real" is turned upside down: the emergent process is real, whereas the process involving "basic" elements is only apparent since it is mediated by the upper level. This means ontological emergence in the strongest sense of the word: the only real process is the high level one. But of course, several difficult questions arise at this point. Let me develop two of them.

The first question concerns the implementation of the abstract idea of "fusion" on a quantum theoretical substrate. Is "fusion" of features or even entities (which are construed as nothing else than quantized features of a field)

\footnotetext{
${ }^{11}$ F.M. Kronz \& J. T. Tiehen, “ Emergence and Quantum Mechanics ”, loc. cit.

${ }^{12}$ P. Humphreys, “ How Properties Emerge”, loc. cit.
} 
a faithful translation in ordinary language and concepts of the non-separability of state vectors or Hamiltonians? Do quantum theories support literally the statement that constituents somehow disappear within a holistic entity? This very strong claim has been overtly challenged, but in a way that preserves the former relation between ontological emergence and quantum physics. On the one hand, no quantum formalism (neither Quantum Mechanics nor Quantum Field Theory) substantiates the idea of a complete disappearance of the many parts within the whole. In standard Quantum Mechanics, the parts still play a role as a set of labeled subsystems mutually correlated by the rules of symmetrization (Bosons) or antisymmetrization (Fermions). And in Quantum Field Theory, the observable "Number" takes on values which are usually greater than one. An order of multiplicity persists in spite of the holistic feature of non-separability. Yet, on the other hand, the parts are usually indistinguishable. They have an identical function within the overall system, and they cannot thus be identified individually with some element which existed prior to the compound system. To sum up, in the quantum paradigm, there is a mutual relation of co-arising between the whole and its parts. The many parts are still taken as constitutive of the whole, but at the same time the whole is irretrievably involved in the definition of the parts since no independent characterization of each sub-system can be given. This remark on the quantum theory of wholes and parts gave rise to a conception called "dynamical emergence" ${ }^{13}$. It represents a middle path between "radical emergence" (the fusion view) and prototypical emergence (the usual view of pre-existing parts). But it mostly retains the strong conception of emergence which is typical of the fusion view. Definition of the parts relative to the whole is as constraining, in this respect, as complete disappearance of the parts within the whole.

The second question concerns the concept of property which, in a quantum theoretical framework, is either inappropriate or completely redefined with respect to its classical and everyday counterpart. The widespread use of the word "property" without qualification by the philosophers of emergence is then utterly misleading, and this challenges virtually every step of their thought process about quantum emergence. Indeed, as the previous paragraphs have shown, the notions of property and property-fusion are taken for granted in the ongoing debate on emergence, with no systematic examination of the very special meaning they must be ascribed in quantum physics. One reads for instance that, in Quantum Mechanics, there exists “(...) a holistic (emergent) correlation property possessed by the system but not locally carried by the separate parts" $"$.

\footnotetext{
${ }^{13}$ F.M. Kronz \& J. T. Tiehen, “ Emergence and Quantum Mechanics ”, loc. cit.

${ }^{14}$ Michael Silberstein \& John McGeever, "The Search for Ontological Emergence”, The philosophical Quarterly, 49, 182-200, 1999
} 
There are only two types of indications that a deep problem arises at this point, but they remain very cryptic.

The first type of indication is provided by a few final statements such as: "One might be hesitant to use quantum entanglements as an argument by themselves because of the notorious difficulties involved in providing a realist interpretation for the theory" ${ }^{15}$. But doubts about the possibility of finding "a realist interpretation for the theory" are tantamount to doubts about the relevance of the concept of property (of an inherently existing feature) when it is applied to quantum systems. This should have a deep retrospective impact on the vocabulary and presuppositions of the main argument; so much so that it is an open question whether much of this argument would still hold.

Another type of indication consists of a systematic shift of the meaning of the word "property". Whereas in classical physics "properties" were values of variables ascribed to systems, they become associated to more elaborate kinds of mathematical entities in quantum physics. One reads accordingly that "twoby-two matrices having specific symmetry features represent properties and their evolution"16; or that "Von Neumann's key idea was to associate a quantitative property of a system with a projection operator in the corresponding Hilbert space" ${ }^{\prime 17}$. This latter strategy has the advantage of preserving an apparent lexical continuity between the classical and the quantum paradigm (there are still "systems" endowed with "properties" in the usual vocabulary of quantum physicists, as in the good old days of classical physics). But it is philosophically inaccurate. In Quantum Mechanics, a matrix operating in a Hilbert space only represents an observable, namely a set of possible experimental phenomena obtained with a certain class of experimental devices. It corresponds by no means to some (intrinsically possessed) property. As for a projection operator, it is a mathematical device enabling one to calculate the probability of one of the subsets of the formerly described set of phenomena. It does not express some inherent characteristic of the system.

To recapitulate, in quantum physics, the very formal concept of intrinsically possessed property is threatened, in so far as one cannot go beyond relational phenomena and their correlations. This fact should not be taken as a marginal feature of the final discussion about quantum emergence. It should not be hidden by a formal shift of the meaning of the word "property" either.

But is it possible to drop any talk of properties throughout, when the issue of quantum non-separability is at stake? Anton Zeilinger managed to do so in a quite elegant manner by using an information-theoretic mode of expression. According to him, combined experiments performed on an entangled physical

\footnotetext{
${ }^{15}$ P. Humphreys, “How Properties Emerge ”, loc. cit.

${ }^{16}$ F.M. Kronz \& J. T. Tiehen, “ Emergence and Quantum Mechanics ”, loc. cit.

${ }^{17}$ R. Omnès, Understanding quantum mechanics, Princeton University Press, 1999, p. 104
} 
system provide us with only one bit of information, not with several bits of information, as they would arise from a set of local experiments performed on the individual components of an uncorrelated system. Information (information relative to an experimental context), is involved; not intrinsic properties. In the upward direction, fusion of potential experimental information occurs; not fusion of actual properties. And in the downward direction, disentanglement or decorrelation of the available information by systematic preliminary measurements may take place; not splitting of properties.

The consequences of this reformulation for the issue of quantum emergence are quite important. In the absence of true intrinsic properties at the high level of holistic correlation observables, and at the low level of individual observables as well, it is wrong to assert that Quantum Mechanics displays "ontological" emergence. What emerges is only a new mode of possible cognitive relation between the microscopic environment and the available range of experimental devices. Here again, the concept of ontological emergence cannot be sustained. Quantum mechanics provides no fresh argument in favor of emergence of new levels of being. But the reason why this is so helps us finding a happy end to this apparently sad story of "inherent", "ontological" emergence.

\section{Non-ontological throughout}

To see why this apparently disappointing dismissal of intrinsic emergence is pushing us toward a very positive conclusion after all, let me first repeat a most gloomy question: since one can lend no credit to the idea of "ontological" emergence, including in Quantum Mechanics which was our last hope, does this mean that the reductionists are right? Does this mean that, at the end of the day, there are only the elementary constituents and their lowlevel properties? Of course not! Deconstructing the formal concepts of substance and of property in Quantum Mechanics is precisely as challenging for the reductionist as it is for the supporter of "true" emergence of high level intrinsic properties. The physical process may have no substantial roof of emergent properties, it has no substantial ground of elementary properties either, according to the most straightforward reading of Quantum Mechanics. Actually, this claim of groundlessness is presently creeping in many other branches of physics, besides ordinary Quantum Mechanics. In modern cosmology, it has recently given rise to the idea that the observable features of the "elementary" particles are just as much determined by global features of the universe than the other way around ${ }^{18}$. In Quantum Field Theory, the standard model of particle physics is often construed as a mere "effective field

\footnotetext{
18 " The top-down universe", New Scientist, 175, 28, 2002. Forerunners of this view were A. Eddington and E. Schrödinger, respectively in 1936 and 1939. See M. Bitbol, Schrödinger's Philosophy of Quantum Mechanics, Kluwer, 1996
} 


\section{2}

theory" of an underlying model. But this underlying model is likely to be no more "basic" as the standard model itself. Some authors noticed that it could itself easily be construed as the effective field theory of an even deeper model, etc. with no ultimate "basic" layer" ${ }^{19}$. This status of the standard model was taken as a good reason to defend an emergentist position in physics against reductionism, starting from explicitly non-substantialist and non-ontological premises $^{20}$. The "never ending tower" of autonomous domains in Quantum Field Theory indeed concerns domains of study, domains of concepts, but not domains of being.

A more detailed way of entering this new trend of thought, which combines emergentism with non-substantialism, consists in criticizing a very widespread distinction between "basic" and "emergent" features instituted on the ground that emergent features are essentially relational whereas basic properties are monadic. T.W. Deacon ${ }^{21}$, for instance, makes an extensive, subtle, and interesting (though disputable), use of this alleged difference. When he asks "What emerges?", his "(...) answer is not some 'thing' but rather something like a form or pattern or function". The features that "really" emerge are collective "topologies" or "configurations". They are essentially configurational, in so far as they express a definite web of relations between the basic elements. This enables one both to endow emerging features with some sort of autonomy at the purely topological or relational level, and to retain the "fundamental" status of the basic constituants of the world. The topological or relational autonomy manifests itself by the ability of high-level patterns or topological configurations to cause alterations of other patterns and topological configurations. But besides this, the basic constituents are (implicitly or explicitly) ascribed a very different standing, essentially immune from any action exerted by the above level. If one accepts, with T.W. Deacon, that what constitutes emergence is definitely not "the production of new kinds of substance or physical laws", but only patterns, this suggests by contrast that the basis for emergence is made of substances with intrinsic properties ruled by real physical laws. Expressions like "substrate properties" (as opposed to their relational properties from which emergent behavior arises), or "component assemblies" (out of which collective features emerge by mutual cancellations of certain effects and topological amplification of other effects in the "components") confirm this highly dissymmetrical construal of the low and high levels.

\footnotetext{
${ }^{19}$ T.Y. Cao \& S.S. Schweber, “ The conceptual foundations and the philosophical aspects of renormalization theory", Synthese, 97, 33-108, 1993

${ }^{20}$ E. Castellani, “ Reductionism, emergence, and effective field theories ", Studies in History and Philosophy of Modern Physics, 33, 251-267, 2002

${ }^{21}$ T.W. Deacon, “The hierarchic logic of emergence: untangling the interdependence of evolution and selforganization ", loc. cit.
} 
But what if the so-called "substrate properties" happen to be just as much configurational as the high level features? In this case, any dissymmetry between the components and the resulting emergent features would vanish.

This argument was developed by some philosophers who have a good grasp of the situation in contemporary physics. They pointed out that "According to our best science, there are no elementary 'particles' or basic particulars at all (...) What have seemed to be 'particles' are now conceptualized as particle-like processes and interactions resulting from the quantization of field processes and interactions"22. Instead of substantial individuals, Quantum Field Theory deals with types of patterns similar to the familiar vibration modes on violin strings. Furthermore, these patterns themselves can hardly be construed as intrinsic properties of fields but rather, once again, as possible values of contextual observables. Therefore, even from the standpoint of a difference between properties and configurations, there is no essential difference between the alleged "basic" level and the emergent levels. Every level of organization which falls within the domain of study of physics is thoroughly relational. And no level can claim for itself the privilege of being for sure the ultimate one; ultimate and monadic.

Several arguments and reflections thus converge towards the conclusion that the world of physical phenomena is groundless throughout. This latter strong statement obviously does not mean that there exists nothing at all (which would be nihilism). Only that the overall process of which we partake by our actions and cognitive relations has no fundamental level on which everything else rests. It has no absolute fundamental level and no absolute emergent level either, but it has co-emergent order. According to Wittgenstein's beautiful metaphor: "One might almost say that these foundation-walls are carried by the whole house"23.

Along with these remarks, it becomes clear that the difficulties and paradoxes of emergence arise from a desperate attempt at taking a wrong conception of the low level as a model for the high level. If the low level is allegedly made of substantial particles endowed with intrinsic properties, then in order to raise the high level to the same standard of dignity as the low level, one unavoidably asks for true emergent intrinsic properties.

Similarly, if the low level properties are ascribed "causal powers" of their own, in the very strong sense of productive or efficient causality, then one is inclined to ask for causal powers of emergent properties as well. According to the supporters of ontological emergence, it is only this way that one may dispel the epiphenomenalist view of emergent features. This claim is easy to understand. An "epiphenomenon" is by definition a feature that has the same

\footnotetext{
${ }^{22}$ M. Bickhard \& D.T. Campbell, “ Emergence ”, in: P.B. Andersen, C. Emmerche, N.O. Finnemann, \& P.V. Christiansen, Downward causation, Aarhus University Press, 2000; See P. Teller, An interpretive introduction to quantum field theory, Princeton University Press, 1995

${ }^{23}$ L. Wittgenstein, On certainty 248, Basil Blackwell, 1974, p. 33e
} 
14

causes as a physical process, but does not cause by itself any alteration of the latter. An illustration was given by William James after Thomas Huxley. In the epiphenomenalist view, an emergent feature appears to be related to the physical process (or "mechanism") "(...) simply as a collateral product of its working, and to be completely without any power of modifying that working, as the steamwhistle which accompanies the work of a locomotive engine is without influence on its machinery" ${ }^{24}$. Therefore, say the ontological emergentists, if emergent behaviors are to be construed as more than mere epiphenomena with respect to a basic layer of ontological properties, they are bound to be endowed with causal powers; the very same type of causal powers that are ascribed to the basic intrinsic properties. The ontological emergentists thus started a desperate search for "causal powers" where they could only find coarse-grained correlations.

At this point, our philosophical tradition seems to be at the end of the tether. We could then look for some inspiration in another tradition, which we do not have to consider as a paradigm of exceptional insight, but only as a source of genuinely alternative ways of thinking. This inspiration is easily found in the Buddhist Madhyamaka philosophy, which targets "causal power" among the reifications it most strongly dismiss. In the well-known first chapter of his Mûlamadhyamakakârikâ, Nâgârjuna thoroughly criticizes the idea of properties having causal power as part of their essence. However, this is by no means tantamount to dismissing any concept of causality. Another, non-substantial, type of causality plays a major role in the Madhyamaka. In the same chapter, Nâgârjuna thus emphasizes that for any event one may single out conditions acting as its antecedents and formal explanations ${ }^{25}$ Unlike causal powers and caused properties, Conditions and conditioned events are both empty of inherent existence. They are relative both to one another and to a cognitive act. They are mutually connected "(...) neither through absolute existence nor through (absolute) identity" ${ }^{26}$. Yet they are codependently arising and are in turn connected similarly with other events or phenomena.

I think it is exactly this way (this extremely critical and non-ontological way) that we can understand most accurately the processes of emergence.

Consider the crucial case of "downward causation", namely causation from the emergent level to a basic level : from the social to the mental level; from the mental to the biological level; and from the biological to the physical level. Within their predominantly substantialist framework of thought, the emergentists are inclined to ask nothing less than productive causal powers of

\footnotetext{
${ }^{24}$ Quoted by: Owen Flanagan, "Conscious inessentialism and the epiphenomenalist suspicion ", in: N. Block, O. Flanagan, \& G. Guzeldere (eds.), The nature of consciousness, MIT Press, 1998

${ }^{25} \mathrm{~J}$. Garfield, The fundamental wisdom of the middle way (Nagarjuna's Mulamadhyamakakarika), Oxford University Press, 1995

${ }^{26}$ J. Garfield, op.cit., p. 105
} 


\section{5}

the emergent properties on the basic properties. And nothing of the sort is in sight. At most, one can find ways of seing some complex mutual interactions of large numbers of basic components as "trans-scale" causation. For instance, recurrence of massively parallel processes involving cancellation of local phenomena and recursive amplification of collective effects can be "(...) described as (...) circles of causality that extend across levels of scale" 27 . But, here as in the case of properties to be seen as or to be (conveniently) described as is not the same as being, full stop. Substantialism about causation pushes emergentism toward the same dead end as substantialism about entities and properties did.

Now, let me remind the solution we found when the issue of entities and properties was at stake. If the whole process is groundless throughout, then there may be emergence without emergent properties. Not asymmetric emergence of high-level properties out of basic properties, but symmetrical co-emergence of microscopic low-level features and high level behavior. Not emergence of large scale absolute properties out of small scale absolute properties, but co-relative emergence of phenomena. These phenomena, in turn, are to be construed as relative to a certain experimental context, with no possibility of separating them from this context. The notion of emergence thus gains credibility at the very same time it loses ontological content.

\section{Non-ontological causation}

In the same way, there may be inter-relatedness without efficient causality ; and there may be upward and downward causation without any causal power. In a non-substantialist framework of thought, the issue of downward causation is not one of inherent powers, but one of relations and actions. Here, the most relevant concept of causality is not the old aristotelian notion of efficient cause. It is not the strong claim that there exists power in property A to produce property B. It is rather the so-called "interventionist" concept of cause which was elaborated by the Wittgensteinean philosopher Georg Henrik Von Wright ${ }^{28}$. According to the latter (who takes "action" as a primitive concept), configuration $\mathrm{A}$ is a cause of the distinct configuration $\mathrm{B}$ if : (i) whenever A has been actively set up, B occurs with probability p ; (ii) whenever A has been actively removed, B does not occur. Both conditions are necessary, because each one of them serves to exclude a certain type of parasitic effect. Condition (ii) is obviously indispensible, because if after having prevented A, B does not occur, this excludes that the frequent association of A and B was purely fortuitous. But condition (i) is also necessary, because if actively producing A by any means is enough to trigger the appearance of $\mathrm{B}$ (with probability $\mathrm{p}$ ), this excludes that the observed

\footnotetext{
${ }^{27}$ T.W. Deacon, " The hierarchic logic of emergence: untangling the interdependence of evolution and selforganization ", loc. cit.

${ }^{28}$ G.H. Von Wright, Causality and determinism, Columbia University Press, 1974
} 
association of A and B was due to some common cause. Condition (ii) is usually not sufficient for this latter purpose since there are situations where preventing A can only be done at the cost of preventing the common cause of $\mathrm{A}$ and $\mathrm{B}$ to occur.

With this definition in mind, we are entitled to speak of "downward causation" in the following circumstances : if (i) whenever a high-level antecedent has been actively set up by means of a high-level coarse-grained device, a certain group of low-level phenomena, relative to a certain type of fine-grained experimental device, occurs with probability p; (ii) whenever the former high-level antecedent has been actively prevented, the former group of low-level phenomena is not observed.

Since this procedure can easily been turned upside down, downward causation is the symmetric mirror image of upward causation. This strongly constrasts with the substantialist view in which the ontological statuses of the high and low level could not be permuted, thus favoring an asymmetric view of causation, with real upward causation and apparent (or inexistent) downward causation. Here, in a non-substantialist framework of thought, we clearly do not have to make any supposition about the essence or the intrinsic nature of the two levels. It is enough to know how to act selectively at one of the two levels, in order to modify what is observed at the other level. It is no longer question of own-being, but only of endless processes of which we partake by our actions.

The most obvious reductionist objection against downward causation is automatically defused by such a symmetric construal of the high and the low level. This objection runs thus: "When you claim that the cause occurs at the higher level, or even when you say your practice aims at modifying the events at the higher level, aren't you speaking of something which in reality occurs or is acted upon at the atomic level? Isn't your allegedly 'high level' action tantamount to a complex interaction between a bunch of atoms (the object of your intervention) and another bunch of atoms (your body or your instruments)?"29. The argument would be conclusive if one accepted that the features of the microscopic world reflect the intrinsic properties of preexisting entities (say the atoms or the particles). But if one rather considers that microscopic phenomena, including the outcome of the measurement of spatial observables such as, say, the appearance of atoms by a scanning tunneling microscope, are the emergent byproduct of an instrumental intervention, then the whole perspective is changed. In this case, what appears, and what is acted upon as well, is relative to the instrument one uses. The scale of the action is defined by the resolution of the tool which has been selected for that task, not by any pre-existing constituents of both the object

\footnotetext{
${ }^{29}$ I thank David Rudrauf (LENA, Hôpital de la Salpêtrière, Paris) for formulating this sound reductionist objection.
} 


\section{7}

and the tool. Therefore, whenever a low resolution tool is used to act upon a macroscopic process, it cannot be said that what one is really doing is acting on the atomic elements of this process. One literally acts at a high, emergent, level by using such a tool, in spite of the fact that other instruments (at much higher resolution) would counterfactually induce atom-like probing and atomlike phenomena if they were used instead.

This trend of thought can be reinforced by pointing out that the authors of the objection are wrongly supposing that the byproduct of investigations in microphysics have a kind of logical and ontological priority over the features of our macrophysical environment. Ascribing this priority is wrong because, as Bohr taught us, the concepts of macrophysics are exactly as indispensible to characterize microphenomena as the concepts of microphysics are indispensible to explain certain macrophenomena. In the quantum paradigm, the relation between the microscopic and macroscopic domains is not dissymmetrical; it is not a mereological part-aggregate type of relation. It is as fully symmetrical as one may conceive: phenomena and concepts relevant at the macrolevel are just as much constitutive of the microlevel as the elements of the microlevel are taken to be constitutive (yet in another sense) of the macrolevel.

\section{Conclusion}

The whole issue of ontological versus epistemological emergence is overcome thus.

Remind what the alternative was. The emerging properties could only have two statuses: either they were intrinsically existent properties (as the low level properties were supposed to be), or they were only fleeting appearances, produced by the coarse instuments we use in order to know complex systems. Either they were endowed with causal power (as the low level properties were supposed to be) or they were only epiphenomena with no causal relevance whatsoever.

But here, both low level and high level features are true processes yet not intrinsically existent. Both are causally relevant to one another, yet not in any one-directional metaphysical sense. Last but not least, both arise from a relation with a cognitive structure, yet are not merely apparent. But how can it be so? Remind that the condition for a distinction between appearance and reality to make sense is the possibility of detachment from the cognitive relation. Appearance is what is relative to some given state of the cognitive apparatus, whereas reality is what has been proven to be invariant with respect to changes of cognitive standpoint. Now, suppose that no such method for detachment is available any longer. Or at least, as it is the case in Quantum Physics which is our current universal theoretical framework, that the only extractable invariant is statistical rather that individual, dispositional rather than actual, or probabilistically predictive rather than phenomenally effective. 
Then, there is no true gap left, at the level of actuality, between Being and Appearance. There is nothing to oppose to relative phenomena, nothing to constrast them, and thus no reason to claim that they are only apparent. The entire framework of subject-object dualism has been short-circuited thus.

Of course, in order to be self-consistent, such a conception must not claim to provide us with some absolute truth about the ultimate nature of elementary and emergent features (be it relational, processual or participatory). It is useful enough if it proves to be an efficient cure against the conceptual rigidities which give rise to dead-end issues, such as the problem of "ontological emergence"

\section{Bibliography}

Bickhard, M. and Campbell, D.T. Emergence. in: P.B. Andersen, C. Emmerche, N.O. Finnemann, and P.V. Christiansen (eds.), Downward causation, Aarhus : Aarhus University Press, 2000

Bitbol, M. 1996. Schrödinger's Philosophy of Quantum Mechanics, Dortrecht : Kluwer

Bitbol, M. 2000. Physique et Philosophie de l'Esprit, Paris : Flammarion

Bitbol, M. 2002. Science as if situation mattered. Phenomenology and the Cognitive Science, 1, 181-224

Cao, T.Y. and Schweber, S.S. 1993. The conceptual foundations and the philosophical aspects of renormalization theory. Synthese, 97, 33-108

Castellani, E. 2002. "Reductionism, emergence, and effective field theories", Studies in History and Philosophy of Modern Physics, 33, 251-267

Deacon, T.W. 2003. The hierarchic logic of emergence: untangling the interdependence of evolution and self-organization. in: B. Weber \& D. Depew (eds.), Evolution and learning: The Baldwin effect reconsidered, Cambridge : MIT Press

Flanagan, O. 1998. Conscious inessentialism and the epiphenomenalist suspicion. in: N. Block, O. Flanagan, \& G. Guzeldere (eds.), The nature of consciousness, Cambridge : MIT Press, 1998

Freeman, A. (ed.) 2001. The emergence of consciousness, Thorverton: Imprint Academic 
19

Garfield, J. 1995. The fundamental wisdom of the middle way (Nagarjuna's Mulamadhyamakakarika), New-York : Oxford University Press

Humphreys, P. 1997. How Properties Emerge, Philosophy of Science, 64, 1-17

Kronz, F.M. and Tiehen, J. T. 2002. Emergence and Quantum Mechanics. Philosophy of Science, 69, 324-347

Lloyd Morgan, C. 1923. Emergent evolution, London: Williams and Norgate

Minkel, J.R. 2002. The top-down universe. New Scientist, 175, 28

Nietzsche, F. 1956. Wille zur Macht, Stuttgart : Alfred Kröner

Omnès, R. 1999. Understanding quantum mechanics, Princeton : Princeton University Press

Schrödinger, E. 1935. The Present Situation of Quantum Mechanics. English Translation in : J.A. Wheeler and W.H. Zurek, (eds.), Quantum Theory and Measurement, Princeton : Princeton university Press, 1983.

Silberstein, M. and McGeever, J. 1999. The Search for Ontological Emergence. The philosophical Quarterly, 49, 182-200

Teller, P. 1995. An interpretive introduction to quantum field theory, Princeton : Princeton University Press

Von Wright, G.H. 1974. Causality and determinism, New-York : Columbia University Press

Wittgenstein, L. 1974. On certainty, Oxford : Basil Blackwell 